\title{
Globally Asymptotic Stability of Stochastic Nonlinear Systems by the Output Feedback
}

\author{
Wenwen Cheng, ${ }^{1}$ Quanxin Zhu, ${ }^{1}$ and Zhangsong Yao ${ }^{2}$ \\ ${ }^{1}$ School of Mathematical Sciences and Institute of Finance and Statistics, Nanjing Normal University, Nanjing, Jiangsu 210023, China \\ ${ }^{2}$ School of Information Engineering, Nanjing Xiaozhuang University, Nanjing, Jiangsu 211171, China \\ Correspondence should be addressed to Zhangsong Yao; yaozhsong@163.com
}

Received 29 March 2015; Revised 31 May 2015; Accepted 7 June 2015

Academic Editor: Antonino Laudani

Copyright (c) 2015 Wenwen Cheng et al. This is an open access article distributed under the Creative Commons Attribution License, which permits unrestricted use, distribution, and reproduction in any medium, provided the original work is properly cited.

\begin{abstract}
We address the problem of the globally asymptotic stability for a class of stochastic nonlinear systems with the output feedback control. By using the backstepping design method, a novel dynamic output feedback controller is designed to ensure that the stochastic nonlinear closed-loop system is globally asymptotically stable in probability. Our way is different from the traditional mathematical induction method. Indeed, we develop a new method to study the globally asymptotic stability by introducing a series of specific inequalities. Moreover, an example and its simulations are given to illustrate the theoretical result.
\end{abstract}

\section{Introduction}

As is well known, the stability problem of nonlinear systems with the state feedback or output feedback control has received much attention since it can be extensively applied in many fields such as engineering and finance. In the practical application, nonlinear systems with the feedback control can model many kinds of stochastic influences either natural or man-made. The output feedback control especially has been used more widely for the reason that a system by the output feedback is more flexible to respond to the information of control systems than the state feedback.

In recent years, there has been a larger number of research works on the global stability for nonlinear systems with the output feedback control [1-6]. For example, Qian and Lin [5] have considered the global stability by the output feedback for a family of triangular nonlinear systems in which the gain parameter $L$ is dependent on the parameters of the controller. Combining the backstepping method and output feedback domination approach, M.-L. Liu and Y.-G. Liu [4] have investigated the semiglobally asymptotic stability for a class of uncertain nonlinear systems. In [1], Andrieu and Praly have applied the output feedback to study the globally asymptotic stability of nonlinear systems based on a unifying point. In [2], Du et al. have discussed the global output feedback stabilization of a class of uncertain upper-triangular systems with the input delay in which the controller with a scaling gain was used to deal with a larger input delay by a coordinate change. However, all the above works did not consider noise disturbances. Actually, the synaptic transmission in real systems can be viewed as a noisy process introduced by random fluctuations from the release of information and other probabilistic causes. Moreover, a system can be stabilized or destabilized by certain stochastic inputs. Therefore, noise disturbances should be taken into account when studying the stability of nonlinear systems.

It is worth pointing out that the problem of global output feedback stability for a class of deterministic lowertriangular systems has been solved in [5] by using the feedback domination design method and constructing a linear output compensator. Unfortunately, the noise disturbance was ignored in [5]. As discussed in the above, the noise disturbance has an important effect on the stability of a real system. So it is natural to question whether a nonlinear output feedback system is stable or not when it is affected by the noise disturbance. About this issue, the previous work on output feedback control of stochastic nonlinear systems almost combines the backstepping method and the mathematical induction to design the output feedback control. For example, Liu et al. $[7,8]$ have discussed the output feedback 
control of a class of stochastic nonlinear systems with linearly bounded unmeasurable states and a class of stochastic nonminimum-phase nonlinear systems. Chen et al. [9] and Liu and Xie [10] have talked about the state feedback stability for stochastic nonlinear systems with time-varying delay. Guo et al. [11] have solved the output feedback stability for a class of stochastic nonlinear systems with power growth conditions. More results can be found in [12-16]. The proofs in these papers are complicated.

In the spirit of stochastic stability theorem of Khasminskii [17] and that of Mao [18] about globally asymptotic stability in probability, we construct a novel Lyapunov function directly to prove the stability of the nonlinear stochastic output feedback system. As the discussion in [5], we also abandon the separation principle paradigm and apply a recursive control algorithm to design the linear control and the Lyapunov function. Different from the mathematical induction, we use some of the ingenious distortion of inequalities to make the infinitesimal generator negative definite. To obtain more concise result, we take the dynamic gain from 2 rather than 1 . In particular, a novel linear observer system is designed and the Lyapunov function is constructed by the following formula:

$$
V=\frac{n+1}{2}\left(\varepsilon^{T} P \varepsilon\right)^{2}+\sum_{j=1}^{n} \frac{1}{4 L^{4 j-4}} \xi_{j}^{4}
$$

Without using the mathematical induction, we construct some variables to achieve the multiform inequalities. As a consequence, our result has more brief frame of the linear controller than that given in [19]. Moreover, the model discussed in [19] can be regarded as the special case of ours.

The rest of this paper is arranged as follows. In Section 2, we present the preparation of globally asymptotic stability and introduce some inequalities which play an important role in the proof of our main results. In Section 3, a novel dynamic output feedback is designed by the backstepping procedure. In Section 4, we use an example to illustrate the theoretical results. Finally, in Section 5, we conclude the paper with some general remarks.

\section{Preliminaries}

In this section, we mainly give the definition of the globally asymptotic stability in probability and introduce several preliminary lemmas.

Consider the following stochastic nonlinear systems:

$$
\begin{aligned}
d x & =f(x) d t+g(x) d \omega, \\
x(0) & =x_{0} \in \mathbb{R}^{n},
\end{aligned}
$$

where $x \in \mathbb{R}^{n}$ is the state; $\omega$ is an $r$-dimensional standard Brownian motion; and the Borel measurable functions $f$ : $\mathbb{R}^{n} \rightarrow \mathbb{R}^{n}$ and $g: \mathbb{R}^{n} \rightarrow \mathbb{R}^{n \times r}$ are locally Lipschitz and satisfy $f(0)=0, g(0)=0$.

Definition 1. The function $\gamma(\cdot): \mathbb{R}_{+} \rightarrow \mathbb{R}_{+}$is said to be $\mathscr{K}$, if $\gamma(\cdot)$ is continuous, strictly increasing, and vanishing at zero.
Definition 2 (see [8]). The equilibrium $x=0$ of (2) is said to be globally asymptotically stable in probability if, for any $\varepsilon>0$, there exists $\gamma(\cdot) \in \mathscr{K}$ such that

$$
P\left\{|x(t)|<\gamma\left(\left|x_{0}\right|\right)\right\} \geq 1-\varepsilon, \quad \forall t \geq 0, \quad x_{0} \in \mathbb{R}^{n} \backslash\{0\},
$$

and for any initial condition $x_{0}$,

$$
P\left\{\lim _{t \rightarrow \infty} x(t)=0\right\}=1
$$

Definition 3 (see [8]). For any given $V(x) \in \mathscr{C}^{2}$ associated with system (2), the differential operator $\mathscr{L}$ is defined as $\mathscr{L} V=\left(\partial V / \partial x^{T}\right) f+(1 / 2) \operatorname{tr}\left\{g^{T}\left(\partial^{2} V / \partial x^{2}\right) g\right\}$, where $(1 / 2) \operatorname{tr}\left\{g^{T}\left(\partial^{2} V / \partial x^{2}\right) g\right\}$ is called Hessian term of $\mathscr{L}$.

Lemma 4 (see [8]). Consider system (2) and suppose that there exist positive definite, radially unbounded, twice continuously differentiable function $V(x)$, and a positive definite function $W(x)$ such that $\mathscr{L} V(x) \leq-W(x)$; then

(i) for (2) there exists an almost surely unique strong solution on $[0, \infty)$ for each $x_{0} \in \mathbb{R}^{n}$;

(ii) the equilibrium $x=0$ of system (2) is globally asymptotically stable in probability.

Lemma 5 (see [13]). Let $p \geq 1$. Then for any $x, y \in \mathbb{R}$,

$$
\begin{aligned}
\left|x^{1 / p}-y^{1 / p}\right| & \leq 2^{(p-1) / p}|x-y|^{1 / p} \\
|x \pm y|^{p} & \leq 2^{p-1}\left|x^{p} \pm y^{p}\right| \leq 2^{p-1}\left(\left|x^{p}\right|+\left|y^{p}\right|\right) \\
(|x|+|y|)^{1 / p} & \leq|x|^{1 / p}+|y|^{1 / p} \leq 2^{(p-1) / p}(|x|+|y|)^{1 / p} .
\end{aligned}
$$

Lemma 6 (see [13]). For any given real numbers $c$, $d$ and any real-valued functions $f(x, y)>0, g(x, y, z) \geq 0$, the following inequality holds:

$$
\begin{aligned}
g(x, y, z)|x|^{c}|y|^{d} \\
\leq \frac{c}{c+d} f(x, y)|x|^{c+d}+\frac{d}{c+d} \\
\quad \times(g(x, y, z))^{(c+d) / d}(f(x, y))^{-c / d}|y|^{c+d},
\end{aligned}
$$

where $x, y, z \in \mathbb{R}$. Particularly when one takes $f(x, y)=$ $g(x, y, z)=1, c=3$, and $d=4$, then the inequality will become

$$
x^{3} y \leq \frac{3}{4} x^{4}+\frac{1}{4} y^{4}
$$

Lemma 7. For any constants $a>0$ and $b \in \mathbb{R}$, one has that, for any $x, y \in \mathbb{R}$,

$$
\begin{aligned}
& -a x^{4}+b x y^{3} \leq k_{1} a^{-1 / 3} b^{4 / 3} y^{4} \\
& -a x^{4}+b x^{3} y \leq k_{2} b^{4} a^{-3} y^{4}
\end{aligned}
$$

where $k_{1}=4^{-1 / 3}-4^{-4 / 3}>0$ and $k_{2}=(3 / 4)^{3}-(3 / 4)^{4}>0$. 
Proof. We first prove (8). Let $Z_{1}(x)=-a x^{4}+b x y^{3}$, where $y$ is a parameter. Then we have

$$
Z_{1}^{\prime}(x)=-4 a x^{3}+b y^{3}=0, \quad x=\left(\frac{b}{4 a}\right)^{1 / 3} y .
$$

So for any $x \in\left(-\infty,(b / 4 a)^{1 / 3} y\right), Z_{1}^{\prime}(x)>0$, and $x \in$ $\left((b / 4 a)^{1 / 3} y,+\infty\right), Z_{1}^{\prime}(x)<0$. With the sufficient condition of extreme value, $x=(b / 4 a)^{1 / 3} y$ is the maximum point of function $Z_{1}(x)$. Therefore, it follows that

$$
\begin{aligned}
Z_{1}(x) & \leq-a\left[\left(\frac{b}{4 a}\right)^{1 / 3} y\right]^{4}+b\left[\left(\frac{b}{4 a}\right)^{1 / 3} y\right] y^{3} \\
& =\left(4^{-1 / 3}-4^{-4 / 3}\right) a^{-1 / 3} b^{4 / 3} y^{4} .
\end{aligned}
$$

We now prove (9). Similarly, letting $Z_{2}(x)=-a x^{4}+b x^{3} y$, where $y$ is a parameter, we have

$$
Z_{2}^{\prime}(x)=-4 a x^{3}+3 b x^{2} y=0, \quad x=\frac{3 b y}{4 a} \text { or } x=0 .
$$

So for any $x \in(-\infty, 3 b y / 4 a), Z_{2}^{\prime}(x)>0$, and $x \in(3 b y /$ $4 a,+\infty), Z_{2}^{\prime}(x)<0$. With the sufficient condition of extreme value, $x=3 b y / 4 a$ is the maximum point of function $Z_{2}(x)$. Thus, we get

$$
\begin{aligned}
Z_{2}(x) & \leq-a\left(\frac{3 b y}{4 a}\right)^{4}+b\left(\frac{3 b y}{4 a}\right)^{3} y \\
& =\left[\left(\frac{3}{4}\right)^{3}-\left(\frac{3}{4}\right)^{4}\right] b^{4} a^{-3} y^{4} .
\end{aligned}
$$

Lemma 8. For a series of numbers $a_{1}, a_{2}, \ldots, a_{n} \in \mathbb{R}$, one has

$$
\begin{aligned}
\left|a_{1}+a_{2}+\cdots+a_{n}\right| & \geq \sqrt{a_{1}^{2}+a_{2}^{2}+\cdots+a_{n}^{2}}, \\
\sqrt{\frac{a_{1}^{2}+a_{2}^{2}+\cdots+a_{n}^{2}}{n}} & \geq \frac{a_{1}+a_{2}+\cdots+a_{n}}{n} \\
& \geq \sqrt[n]{a_{1} a_{2} \cdots a_{n}} \\
& \geq \frac{2}{1 / a_{1}+1 / a_{2}+\cdots+1 / a_{n}} .
\end{aligned}
$$

Lemma 9 (Cauchy-Schwartz's inequality). Let the vector $x=$ $\left(x_{1}, x_{2}, \ldots, x_{n}\right) \in \mathbb{R}^{n}$ and $y=\left(y_{1}, y_{2}, \ldots, y_{n}\right) \in \mathbb{R}^{n}$, then

$$
\begin{aligned}
& \left(x_{1} y_{1}+x_{2} y_{2}+\cdots+x_{n} y_{n}\right)^{2} \\
& \quad \leq\left(x_{1}^{2}+\cdots+x_{n}^{2}\right)\left(y_{1}^{2}+\cdots+y_{n}^{2}\right) .
\end{aligned}
$$

Lemma 10 (Young's inequality). For vectors $x \in \mathbb{R}^{n}, y \in \mathbb{R}^{n}$, one has $x^{T} y \leq\left(\varepsilon^{p} / p\right)|x|^{p}+\left(1 / q \varepsilon^{q}\right)|y|^{q}$, where $\varepsilon>0, p>1$, $q>1$, and $1 / p+1 / q=1$.

\section{The Output Feedback Model and Control Design}

In this section, we design a novel linear observer system (18) for the stochastic nonlinear system (16) below. Using the backstepping method, a simple linear control is constructed to guarantee that the closed-loop stochastic system is globally asymptotically stable in probability.

Consider the following nonlinear stochastic system:

$$
\begin{gathered}
d x_{1}=\left(x_{2}+f_{1}\left(\bar{x}_{1}\right)\right) d t+g_{1}^{T}\left(\bar{x}_{1}\right) d w, \\
d x_{2}=\left(x_{3}+f_{2}\left(\bar{x}_{2}\right)\right) d t+g_{2}^{T}\left(\bar{x}_{2}\right) d w, \\
\vdots \\
d x_{n-1}=\left(x_{n}+f_{n-1}\left(\bar{x}_{n-1}\right)\right) d t+g_{n-1}^{T}\left(\bar{x}_{n-1}\right) d w, \\
d x_{n}=\left(u+f_{n}\left(\bar{x}_{n}\right)\right) d t+g_{n}^{T}\left(\bar{x}_{n}\right) d w, \\
y=x_{1},
\end{gathered}
$$

where $\bar{x}_{i}=\left(x_{1}, \ldots, x_{i}\right)$ is the state vector, $u \in \mathbb{R}$ is the control input, $w$ is the $r$-dimensional standard Wiener process, and $y \in \mathbb{R}$ are the system output. The nonlinear functions $f_{i}$ : $\mathbb{R}_{+} \times \mathbb{R}^{i} \rightarrow \mathbb{R}$ and $g_{i}: \mathbb{R}_{+} \times \mathbb{R}^{i} \rightarrow \mathbb{R}^{r}$ are locally Lipschitz with $f_{i}(0)=0, g_{i}(0)=0, i=1, \ldots, n$.

Assumption 11. The nonlinear functions $f_{i}\left(\bar{x}_{i}\right) \in \mathbb{R}$ and $g_{i}\left(\bar{x}_{i}\right) \in \mathbb{R}^{r}, i=1, \ldots, n$, are locally Lipschitz with $f_{i}(0)=0$ and $g_{i}(0)=0$ for $i=1, \ldots, n$. Moreover, there exist two constants $l_{1} \geq 0$ and $l_{2} \geq 0$ such that

$$
\begin{aligned}
& \left|f_{i}\left(\bar{x}_{i}\right)\right| \leq l_{1}\left(\left|x_{1}\right|+\cdots+\left|x_{i}\right|\right), \\
& \left|g_{i}\left(\bar{x}_{i}\right)\right| \leq l_{2}\left(\left|x_{1}\right|+\cdots+\left|x_{i}\right|\right) .
\end{aligned}
$$

The linear observer system is designed as

$$
\begin{gathered}
\dot{\hat{x}}_{1}=\widehat{x}_{2}(t)+L a_{1}\left(x_{1}-\widehat{x}_{1}\right), \\
\dot{\hat{x}}_{2}=\widehat{x}_{3}(t)+L^{2} a_{2}\left(x_{1}-\widehat{x}_{1}\right), \\
\vdots \\
\dot{\hat{x}}_{n-1}=\widehat{x}_{n}(t)+L^{n-1} a_{n-1}\left(x_{1}-\widehat{x}_{1}\right), \\
\dot{\hat{x}}_{n}=u+L^{n} a_{n}\left(x_{1}-\widehat{x}_{1}\right),
\end{gathered}
$$

where $L \geq 2$ is an appropriate constant and $a_{i}>0, i=$ $1, \ldots, n$, are coefficients of the Hurwitz polynomial:

$$
p(t)=t^{n}+a_{1} t^{n-1}+\cdots+a_{n-1} t+a_{n}
$$


The observation error $\varepsilon_{i}=\left(x_{i}-\widehat{x}_{i}\right) / L^{i-1}$ satisfies

$$
\begin{gathered}
d \varepsilon=L\left[\begin{array}{ccccc}
-a_{1} & 1 & 0 & \cdots & 0 \\
-a_{2} & 0 & 1 & \cdots & 0 \\
\vdots & \vdots & \vdots & \ddots & \vdots \\
-a_{n-1} & 0 & 0 & \cdots & 0 \\
-a_{n} & 0 & 0 & \cdots & 0
\end{array}\right] \varepsilon d t+\left[\begin{array}{c}
f_{1} \\
\frac{f_{2}}{L} \\
\vdots \\
\frac{f_{n-1}}{L^{n-2}} \\
\frac{f_{n}}{L^{n-1}}
\end{array}\right] d t \\
+\left[\begin{array}{c}
g_{1}^{T} \\
\frac{g_{2}^{T}}{L} \\
\vdots \\
\frac{g_{n-1}^{T}}{L^{n-2}} \\
\frac{g_{n}^{T}}{L^{n-1}}
\end{array}\right] d w=L A \varepsilon d t+F d t+G d w,
\end{gathered}
$$

where $A$ is a Hurwitz matrix. Therefore, there is a positivedefinite matrix $P=P^{T}>0$ such that

$$
A^{T} P+P A=-I
$$

Theorem 12. Assume that Assumption 11 holds. Then, the equilibrium at origin of the closed-loop nonlinear stochastic system (16) and (18) with the linear controller (31) below is globally asymptotically stable in probability. Furthermore, it follows from Lemma 4 that there exists an almost surely unique strong solution on $[0, \infty)$ for each $x_{0} \in \mathbb{R}^{n}$.

Proof. Consider the following Lyapunov function $V_{0}(\varepsilon)=$ $((n+1) / 2)\left(\varepsilon^{T} P \varepsilon\right)^{2}$. Then, we have

$$
\begin{aligned}
& \mathscr{L} V_{0}=(n+1) \\
& \cdot\left(\left(\varepsilon^{T} P \varepsilon\right)\left(\varepsilon^{T}\left(L A^{T} P+L P A\right) \varepsilon+2 \varepsilon^{T} P F\right)\right. \\
& \left.+\frac{1}{2} \operatorname{tr}\left(G^{T}\left(4 P \varepsilon \varepsilon^{T} P+2 \varepsilon^{T} P \varepsilon P\right) G\right)\right) \leq-(n+1) \\
& \cdot \lambda_{\min } L\|\varepsilon\|^{4}+2(n+1) \varepsilon^{T} P \varepsilon \varepsilon^{T} P F+3(n+1) \\
& \cdot r \sqrt{r}|G|^{2}|P|^{2}\|\varepsilon\|^{2} \leq-(n+1) \lambda_{\min } L\|\varepsilon\|^{4}+2(n \\
& +1) \lambda_{\max }^{2}\|\varepsilon\|^{3}|F|+3(n+1) r \sqrt{r} \lambda_{\max }^{2}|G|^{2}\|\varepsilon\|^{2},
\end{aligned}
$$

where $\lambda_{\min }$ denotes the minimum eigenvalue and $\lambda_{\max }$ is the maximum eigenvalue of the matrix $P$.
It follows from Assumption 11 that

$$
\begin{aligned}
|F| & =\sqrt{f_{1}^{2}+\left(\frac{f_{2}}{L}\right)^{2}+\cdots+\left(\frac{f_{n}}{L^{n-1}}\right)^{2}} \\
& \leq 2 l_{1}\left(\left|x_{1}\right|+\frac{1}{L}\left|x_{2}\right|+\cdots+\frac{1}{L^{n-1}}\left|x_{n}\right|\right), \\
|G| & =\sqrt{g_{1}^{2}+\left(\frac{g_{2}}{L}\right)^{2}+\cdots+\left(\frac{g_{n}}{L^{n-1}}\right)^{2}} \\
& \leq 2 l_{2}\left(\left|x_{1}\right|+\frac{1}{L}\left|x_{2}\right|+\cdots+\frac{1}{L^{n-1}}\left|x_{n}\right|\right) .
\end{aligned}
$$

Recalling that $x_{i}=\widehat{x}_{i}+L^{i-1} \varepsilon_{i}, i=1, \ldots, n$, we get

$$
\begin{aligned}
2( & +1) \lambda_{\max }^{2}\|\varepsilon\|^{3}|F| \leq \frac{3}{2}(n+1) \lambda_{\max }^{2}\|\varepsilon\|^{4} \\
& +\frac{1}{2}(n+1) \lambda_{\max }^{2}|F|^{4} \\
& \leq\left(\frac{3}{2}(n+1)+64(n+1) n^{2} c^{4}\right) \lambda_{\max }^{2}\|\varepsilon\|^{4} \\
& +64(n+1) \\
& \cdot n^{3} c^{4} \lambda_{\max }^{2}\left(\widehat{x}_{1}^{4}+\frac{1}{L^{4}} \widehat{x}_{2}^{4}+\cdots+\frac{1}{L^{4 n-4}} \widehat{x}_{n}^{4}\right) \\
3( & +1) r \sqrt{r} \lambda_{\max }^{2}|G|^{2}\|\varepsilon\|^{2} \leq \frac{3}{2}(n+1) \lambda_{\max }^{2}\|\varepsilon\|^{4} \\
& +\frac{3}{2}(n+1) r^{3} \lambda_{\max }^{2}|G|^{4} \\
& \leq\left(\frac{3}{2}(n+1)+192(n+1) n^{2} r^{3} c^{4}\right) \lambda_{\max }^{2}\|\varepsilon\|^{4} \\
& +192(n+1) n^{3} r^{3} c^{4} \lambda_{\max }^{2}\left(\widehat{x}_{1}^{4}+\cdots+\frac{1}{L^{4 n-4}} \widehat{x}_{n}^{4}\right)
\end{aligned}
$$

where $c=\max \left\{l_{1}, l_{2}\right\}$.

Substituting (24) into (22) yields

$$
\begin{aligned}
\mathscr{L} V_{0} \leq & -\left((n+1) \lambda_{\min } L-c_{0}\right)\|\varepsilon\|^{4} \\
& +c_{1}\left(\widehat{x}_{1}^{4}+\frac{\widehat{x}_{2}^{4}}{L^{4}}+\cdots+\frac{\widehat{x}_{n}^{4}}{L^{4 n-4}}\right),
\end{aligned}
$$

where

$$
\begin{aligned}
& c_{0}=\left(3(n+1)+\left(64+192 r^{3}\right)(n+1) n^{2} c^{4}\right) \lambda_{\max }^{2}, \\
& c_{1}=\left(64+192 r^{3}\right)(n+1) n^{3} c^{4} \lambda_{\max }^{2} .
\end{aligned}
$$

Now, we take the Lyapunov function as follows:

$$
V=\frac{n+1}{2}\left(\varepsilon^{T} P \varepsilon\right)^{2}+\sum_{j=1}^{n} \frac{1}{4 L^{4 j-4}} \xi_{j}^{4} .
$$


And $\widehat{x}_{i}=\xi_{i}+\widehat{x}_{i-1}^{*}, \widehat{x}_{i-1}^{*}=-L b_{i-1} \xi_{i-1}$, and $\widehat{x}_{1}=\xi_{1}$, where $i=2, \ldots, n$ and $b_{i}$ is needed to be determined later. Then, a direct computation yields

$$
\begin{aligned}
& \mathscr{L} V \leq-\left((n+1) \lambda_{\min } L-c_{0}\right)\|\varepsilon\|^{4}+c_{1} \widehat{x}_{1}^{4}+c_{1}\left(\frac{1}{L^{4}} \widehat{x}_{2}^{4}\right. \\
& \left.+\cdots+\frac{1}{L^{4 n-4}} \widehat{x}_{n}^{4}\right)+\xi_{1}^{3}\left(\widehat{x}_{2}+L a_{1} \varepsilon_{1}\right)+\sum_{j=2}^{n-1} \frac{1}{L^{4 j-4}} \xi_{j}^{3} \\
& \cdot\left(\widehat{x}_{j+1}\right. \\
& \left.+L^{j} a_{j} \varepsilon_{1}-\sum_{i=1}^{j-1} \frac{\partial \widehat{x}_{j-1}^{*}}{\partial \widehat{x}_{i}}\left(\widehat{x}_{i+1}+L^{i} a_{i} \varepsilon_{1}\right)\right)+\frac{1}{L^{4 n-4}} \xi_{n}^{3}(u \\
& \left.+L^{n} a_{n} \varepsilon_{1}-\sum_{i=1}^{n-1} \frac{\partial \widehat{x}_{n-1}^{*}}{\partial \widehat{x}_{i}}\left(\widehat{x}_{i+1}+L^{i} a_{i} \varepsilon_{1}\right)\right) \\
& \leq-\left((n+1) \lambda_{\min } L-c_{0}\right)\|\varepsilon\|^{4}+c_{1} \xi_{1}^{4} \\
& +8 c_{1} \sum_{j=2}^{n} \frac{1}{L^{4 j-4}} \xi_{j}^{4}+8 c_{1} \sum_{j=2}^{n} \frac{b_{j-1}^{4}}{L^{4 j-8}} \xi_{j-1}^{4}+\xi_{1}^{3}\left(-L b_{1} \xi_{1}\right. \\
& \left.+L a_{1} \varepsilon_{1}\right)+\sum_{j=1}^{n-1} \frac{1}{L^{4 j-4}}\left(\xi_{j}^{3} \xi_{j+1}-L \xi_{j}^{4}\right)+\sum_{j=1}^{n-1} \frac{1}{L^{4 j-5}} \xi_{j}^{4} \\
& +\sum_{j=2}^{n-1} \frac{1}{L^{4 j-4}} \xi_{j}^{3}\left(-L b_{j} \xi_{j}\right. \\
& \left.+\left(L^{j} a_{j} \varepsilon_{1}-\sum_{i=1}^{j-1} \frac{\partial \widehat{x}_{j-1}^{*}}{\partial \widehat{x}_{i}} L^{i} a_{i} \varepsilon_{1}\right)-\sum_{i=1}^{j-1} \frac{\partial \widehat{x}_{j-1}^{*}}{\partial \widehat{x}_{i}} \widehat{x}_{i+1}\right) \\
& +\frac{1}{L^{4 n-4}} \xi_{n}^{3}\left(u+L^{n} a_{n} \varepsilon_{1}\right. \\
& \left.-\sum_{i=1}^{n-1} \frac{\partial \widehat{x}_{n-1}^{*}}{\partial \widehat{x}_{i}}\left(\widehat{x}_{i+1}+L^{i} a_{i} \varepsilon_{1}\right)\right) \leq-\left(\lambda_{\min } L-c_{0}\right)\|\varepsilon\|^{4} \\
& +\left(\left(-b_{1}+c_{1}+a_{1}^{\prime}\right) L+8 c_{1} b_{1}^{4}\right) \xi_{1}^{4}+8 c_{1} \sum_{j=3}^{n} \frac{b_{j-1}^{4}}{L^{4 j-8}} \xi_{j-1}^{4} \\
& +\sum_{j=2}^{n} \frac{m_{j-1}}{L^{4 j-5}} \xi_{j}^{4}+\sum_{j=1}^{n} \frac{k_{2}+1}{L^{4 j-5}} \xi_{j}^{4}+\sum_{j=2}^{n-1} \frac{1}{L^{4 j-4}} \xi_{j}^{3}\left(-L b_{j} \xi_{j}\right. \\
& \left.+L b_{j-1} \xi_{j}+L^{2} b_{j-1, j-1} \xi_{j-1}+\cdots+L^{j} b_{j-1,1} \xi_{1}\right) \\
& +\frac{1}{L^{4 n-4}} \xi_{n}^{3}\left(u+L b_{n-1} \xi_{n}+\cdots+L^{n-1} b_{n-1,2} \xi_{2}\right. \\
& \left.+L^{n} b_{n-1,1} \xi_{1}\right)
\end{aligned}
$$

where

$$
\begin{aligned}
& a_{1}^{\prime}=k_{1} \lambda_{\min }^{-1 / 3} a_{1}^{4 / 3}, \\
& b_{j-1,1}=-b_{j-1} b_{j-2} \cdots b_{2} b_{1}^{2},
\end{aligned}
$$

$$
\begin{aligned}
& b_{j-1,2}=-b_{j-1} b_{j-2} \cdots b_{3} b_{2}^{2}+b_{j-1} b_{j-2} \cdots b_{1}, \ldots \\
& b_{j-1, j-2}=-b_{j-1} b_{j-2}^{2}+b_{j-1} b_{j-2}, \\
& b_{j-1, j-1}=-b_{j-1}^{2}+b_{j-1} b_{j-2}, \\
& m_{j-1}=k_{1} \lambda_{\min }^{-1 / 3}\left(a_{j}+b_{j-1} a_{j-1}+b_{j-1} b_{j-2} a_{j-2}+\cdots\right. \\
& \left.+b_{j-1} \cdots b_{1} a_{1}\right)^{4 / 3}, \quad j=2, \ldots, n .
\end{aligned}
$$

From Lemma 7, it follows that

$$
\begin{aligned}
& \mathscr{L} V \leq-\left(\lambda_{\min } L-c_{0}\right)\|\varepsilon\|^{4}+\left(\left(-b_{1}+c_{1}+a_{1}^{\prime}+k_{2}\right.\right. \\
& \text { + 1) } \left.L+8 c_{1} b_{1}^{4}\right) \xi_{1}^{4}+\sum_{j=2}^{n-1} \frac{-b_{j}}{L^{4 j-5}} \xi_{j}^{4}+8 c_{1} \sum_{j=3}^{n} \frac{b_{j-1}^{4}}{L^{4 j-8}} \xi_{j-1}^{4} \\
& +\sum_{j=2}^{n-1} \frac{1}{L^{4 j-4}} \xi_{j}^{3}\left(\left(b_{j-1}+m_{j-1}+k_{2}+1\right) L \xi_{j}\right. \\
& \left.+L^{2} b_{j-1, j-1} \xi_{j-1}+\cdots+L^{j-1} b_{j-1,2} \xi_{2}+L^{j} b_{j-1,1} \xi_{1}\right) \\
& +\frac{1}{L^{4 n-4}} \xi_{n}^{3}\left(u+L\left(b_{n-1}+m_{n-1}+k_{2}+1\right) \xi_{n}\right. \\
& \left.+L^{2} b_{n-1, n-1} \xi_{n-1}+\cdots+L^{n} b_{n-1,1} \xi_{1}\right) \leq-\left(\lambda_{\min } L\right. \\
& \left.-c_{0}\right)\|\varepsilon\|^{4}+\left(\left(-b_{1}+c_{1}+a_{1}^{\prime}+k_{2}+1\right) L+8 c_{1} b_{1}^{4}\right) \xi_{1}^{4} \\
& +8 c_{1} \sum_{j=2}^{n-1} \frac{b_{j}^{4}}{L^{4 j-4}} \xi_{j}^{4}+\sum_{j=2}^{n-1} \frac{-b_{j}+b_{j-1}+m_{j-1}+k_{2}+1}{L^{4 j-5}} \xi_{j}^{4} \\
& +\frac{1}{L^{4 n-4}} \xi_{n}^{3} u+\sum_{j=1}^{n-1} \frac{n-j}{L^{4 j-5}} \xi_{j}^{4}+\sum_{j=2}^{n} \frac{1}{L^{4 j-4}}\left(\left(-L^{5} \xi_{j-1}^{4}\right.\right. \\
& \left.+L^{2} b_{j-1, j-1} \xi_{j-1} \xi_{j}^{3}\right)+\cdots+\left(-L^{4 j-7} \xi_{2}^{4}\right. \\
& \left.\left.+L^{j-1} b_{j-1,2} \xi_{2} \xi_{j}^{3}\right)+\left(-L^{4 j-3} \xi_{1}^{4}+L^{j} b_{j-1,1} \xi_{1} \xi_{j}^{3}\right)\right) \\
& +\frac{1}{L^{4 n-4}} \xi_{n}^{3}\left(u+L\left(b_{n-1}+m_{n-1}+k_{2}+1\right) \xi_{n}\right) \\
& \leq-\left(\lambda_{\min } L-c_{0}\right)\|\varepsilon\|^{4}+\left(\left(-b_{1}+c_{1}+a_{1}^{\prime}+k_{2}+n\right) L\right. \\
& \left.+8 c_{1} b_{1}^{4}\right) \xi_{1}^{4}+8 c_{1} \sum_{j=2}^{n-1} \frac{b_{j}^{4}}{L^{4 j-4}} \xi_{j}^{4}+\sum_{j=2}^{n-1} \frac{1}{L^{4 j-5}}\left(-b_{j}+b_{j-1}\right. \\
& +m_{j-1}+k_{2}+1+n-j+d_{j-1}+d_{j-2}+\cdots+d_{2} \\
& \left.+d_{1}\right) \xi_{j}^{4}+\frac{1}{L^{4 n-4}} \xi_{n}^{3}\left(u+L\left(b_{n-1}+m_{n-1}+k_{2}+1\right.\right. \\
& \left.\left.+r_{n-1}+r_{n-2}+\cdots+r_{2}+r_{1}\right) \xi_{n}\right) \leq-\left(\lambda_{\min } L-c_{0}\right) \\
& \cdot\|\varepsilon\|^{4}-\sum_{j=1}^{n-1} \frac{1}{L^{4 j-4}}\left(L-8 c_{1} b_{j}^{4}\right) \xi_{j}^{4}-\frac{1}{L^{4 n-5}} \xi_{n}^{4},
\end{aligned}
$$


where

$$
\begin{aligned}
d_{1}= & k_{1} b_{j-1,1}^{4 / 3}, \\
d_{2}= & k_{1} b_{j-1,2}^{4 / 3}, \ldots, d_{j-2}=k_{1} b_{j-1, j-2}^{4 / 3}, \\
d_{j-1}= & k_{1} b_{j-1, j-1}^{4 / 3}, \\
r_{1}= & k_{1} b_{n-1,1}^{4 / 3}, \\
r_{2}= & k_{1} b_{n-1,1}^{4 / 3}, \ldots, r_{n-2}=k_{1} b_{n-1, n-2}^{4 / 3}, \\
r_{n-1}= & k_{1} b_{n-1, n-1}^{4 / 3}, \\
b_{1}= & c_{1}+a_{1}^{\prime}+k_{2}+n+1, \\
b_{j}= & b_{j-1}+m_{j-1}+k_{2}+1+n-j+1+d_{j-1}+\cdots \\
& +d_{1}, \quad j=2, \ldots, n-1, \\
u= & -L b_{n} \xi_{n}=-L\left(b_{n-1}+m_{n-1}+k_{2}+2+r_{n-1}+r_{n-2}\right. \\
& \left.+\cdots+r_{2}+r_{1}\right) \xi_{n} .
\end{aligned}
$$

Now, we choose the gain constant $L=\max \left\{2, c_{0} / \lambda_{\text {min }}, 8 c_{1} b_{1}^{4}\right.$, $\left.\ldots, 8 c_{1} b_{n-1}^{4}\right\}$, and then the right-hand side of (30) becomes negative definite. Therefore, it follows from Lemma 4 that the equilibrium of the closed-loop nonlinear stochastic system is globally asymptotically stable in probability and there exists an almost surely unique strong solution on $[0, \infty)$ for each $x_{0} \in \mathbb{R}^{n}$.

Remark 13. Letting $g_{i}(x) \equiv 0$ for all $x \in \mathbb{R}^{n}$ and $i=$ $1, \ldots, n$, then system (16) is reduced to the deterministic system, which was studied by Qian and Lin in [5]. Therefore, we extend the conclusion for the deterministic system to the stochastic nonlinear system and construct a novel linear output feedback controller.

Remark 14. Letting $f_{i}(x) \equiv 0$ for all $x \in \mathbb{R}^{n}$ and $i=$ $1, \ldots, n$, then system (16) is reduced to that in [19], which was studied by Deng and Krstić. It is worth pointing out that the output feedback control in [19] is nonlinear, which is very complex. However, our research is based on a novel linear output feedback control. Therefore, our result extends and improves that in [19].

Remark 15. In [7], Liu and Zhang studied the stability of stochastic nonlinear systems with linearly bounded unmeasurable states by the output feedback control. It should be mentioned that the mathematical induction played a key role in the proof of the main result in [7]. However, in this paper, we construct a novel lyapunov function and prove the stability directly without using the mathematical induction, which make the proof more concise and help us construct the linear output feedback controller more easily.

\section{An Example}

In this section, we will use an example to illustrate our main result.

Example 1. Consider the following stochastic nonlinear system:

$$
\begin{aligned}
d x_{1} & =\left(x_{2}+\frac{1}{16} x_{1} \sin x_{2}^{2}\right) d t+\frac{1}{16}\left(2 x_{1}^{2}-x_{1}^{3}\right) d w, \\
d x_{2} & =\left(u+\frac{1}{16}\left|x_{1}+x_{2}\right|\right) d t+\frac{1}{16} \ln \left(1+\left|x_{2}\right|\right) d w, \\
y & =x_{1} .
\end{aligned}
$$

Obviously, the functions $f_{1}, f_{2}, g_{1}, g_{2}$ are locally Lipschitz such that

$$
\begin{aligned}
& \left|f_{1}(x)\right| \leq \frac{1}{16}\left|x_{1}\right|, \\
& \left|f_{2}(x)\right| \leq \frac{1}{16}\left(\left|x_{1}\right|+\left|x_{2}\right|\right), \\
& \left|g_{1}(x)\right| \leq \frac{1}{16}\left|x_{1}\right|, \\
& \left|g_{2}(x)\right| \leq \frac{1}{16}\left(\left|x_{1}\right|+\left|x_{2}\right|\right),
\end{aligned}
$$

which verifies that Assumption 11 holds. Moreover, it is easy to check that

$$
\begin{aligned}
& |F| \leq \frac{1}{8}\left(\left|x_{1}\right|+\frac{1}{L}\left|x_{2}\right|\right), \\
& |G| \leq \frac{1}{8}\left(\left|x_{1}\right|+\frac{1}{L}\left|x_{2}\right|\right) .
\end{aligned}
$$

The linear observer system is designed as

$$
\begin{aligned}
& d \widehat{x}_{1}=\left(\widehat{x}_{2}+L\left(x_{1}-\widehat{x}_{1}\right)\right) d t, \\
& d \widehat{x}_{2}=\left(u+L^{2}\left(x_{1}-\widehat{x}_{1}\right)\right) d t,
\end{aligned}
$$

with a suitable choice of the parameter $L$. The observation errors $\varepsilon_{1}=x_{1}-\widehat{x}_{1}$ and $\varepsilon_{2}=\left(x_{2}-\widehat{x}_{2}\right) / L$ satisfy

$$
\begin{aligned}
d \varepsilon & =L\left[\begin{array}{ll}
-1 & 1 \\
-1 & 0
\end{array}\right] \varepsilon d t+\left[\begin{array}{l}
f_{1} \\
\frac{f_{2}}{L}
\end{array}\right] d t+\left[\begin{array}{c}
g_{1}^{T}(y) \\
\frac{g_{2}^{T}(y)}{L}
\end{array}\right] d w \\
& =L A \varepsilon d t+F d t+G d w .
\end{aligned}
$$

For the above matrix $A$, there exists a positive-definite matrix $P$ satisfying $A^{T} P+P A=-I$, where

$$
P=\left[\begin{array}{cc}
1 & -\frac{1}{2} \\
-\frac{1}{2} & \frac{3}{2}
\end{array}\right] .
$$

It is easy to get the minimum eigenvalue $\lambda_{\min }=(5-\sqrt{5}) / 4$ and maximum eigenvalue $\lambda_{\max }=(5+\sqrt{5}) / 4$ of the matrix $P$. 


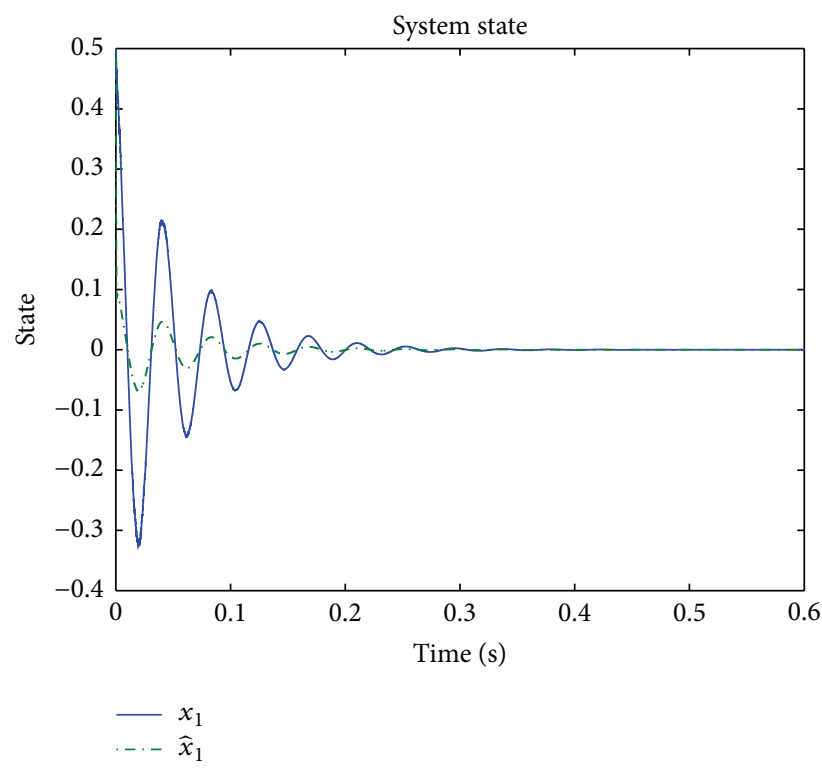

Figure 1: The first state response in Example 1.

Now, taking $V_{0}=(3 / 2)\left(\varepsilon^{T} P \varepsilon\right)^{2}$, then we get

$$
\mathscr{L} V_{0} \leq-\left(3 \lambda_{\min } L-c_{0}\right)\|\varepsilon\|^{4}+c_{1} \hat{x}_{1}^{4}+\frac{c_{1}}{L^{4}} \widehat{x}_{2}^{4},
$$

where

$$
\begin{aligned}
& c_{0}=9.1 \lambda_{\text {max }}^{2}, \\
& c_{1}=0.1 \lambda_{\text {max }}^{2} .
\end{aligned}
$$

Choosing

$$
V=V_{0}+c_{1} \xi_{1}^{4}+\frac{c_{1}}{L^{4}} \xi_{2}^{4},
$$

$\xi_{1}=\widehat{x}_{1}$, and $\xi_{2}=\widehat{x}_{2}+L b_{1} \widehat{x}_{1}$, we have

$$
\mathscr{L} V \leq-\left(\lambda_{\min } L-c_{0}\right)\|\varepsilon\|^{4}+\left(8 c_{1} b_{1}^{4}-L\right) \xi_{1}^{4}-\frac{1}{L^{3}} \xi_{2}^{4},
$$

where $b_{1}=4, b_{2}=29, L=664$, and $u=-L b_{2}\left(\widehat{x}_{2}+L b_{1} \widehat{x}_{1}\right)=$ $-L b_{2} \xi_{2}$. Obviously,

$$
V=\frac{3}{2}\left(\varepsilon^{T} P \varepsilon\right)^{2}+\frac{1}{4} \xi_{1}^{4}+\frac{1}{4 L^{4}} \xi_{2}^{4},
$$

which is positive-definite and proper. By Theorem 12, we see that the equilibrium $x=0$ of the nonlinear closed-loop stochastic system (32) and (35) is globally asymptotically stable in probability and there exist an almost unique strong solution on $[0, \infty)$. The state response and control input with initial conditions $x_{1}=0.5, x_{2}=0, \widehat{x}_{1}=0.6$, and $\widehat{x}_{2}=0$ are presented in Figures 1-3.

Figures 1-3 show that the equilibrium of the closed-loop system is unique and tends to 0 when the initial state is nonzero. In other words, for the closed-loop system, the equilibrium is globally asymptotically stable in probability and there exists an almost surely unique strong solution on $[0, \infty)$ for each $x_{0} \in \mathbb{R}^{n}$, which verifies our theoretical results.

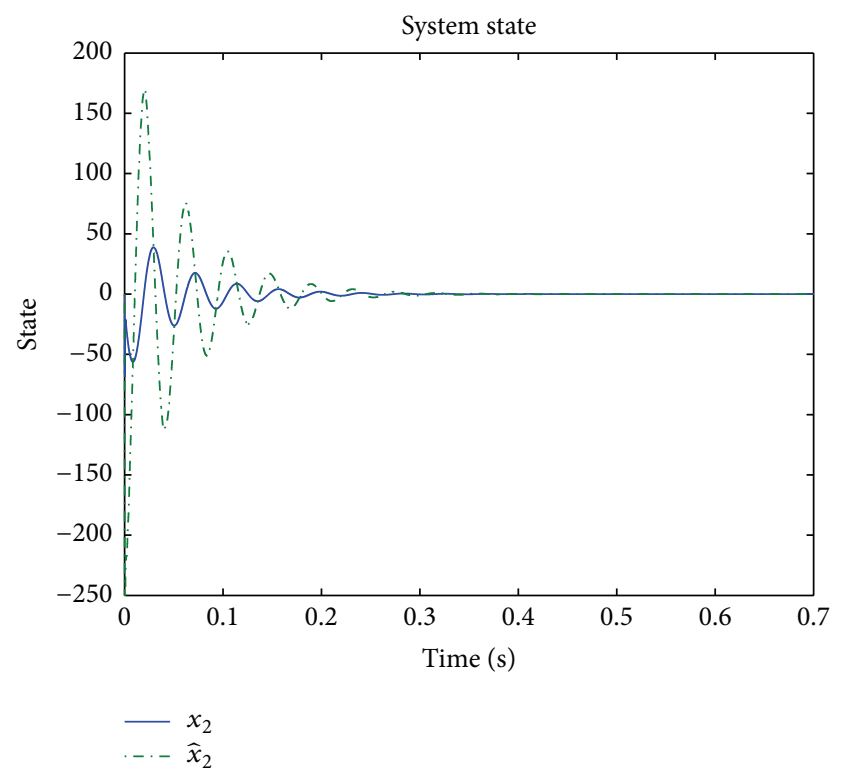

FIGURE 2: The second state response in Example 1.

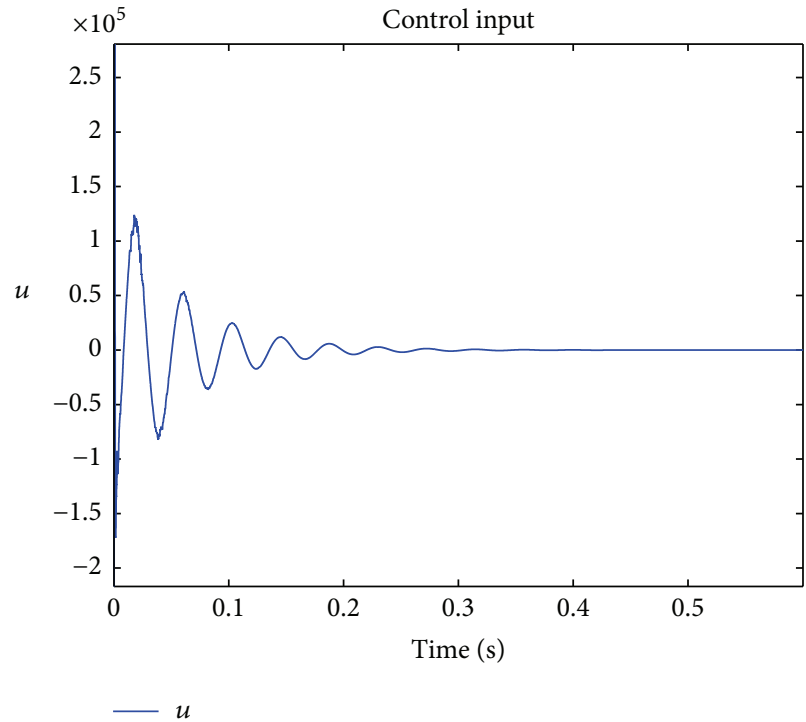

Figure 3: The control input in Example 1.

\section{Conclusion}

In this paper, we have studied the problem of globally asymptotic stability of stochastic nonlinear systems by the output feedback with a novel method. It is worth pointing out that the design of the dynamic output feedback controller plays an important role in the proof of our main result, especially that the Young inequality is a key tool. We believe that our formulation and approach can be used to analyse the stabilization problem of stochastic nonlinear systems with input delays, in which the feedback domination design will be a more complex structure. 


\section{Conflict of Interests}

The authors declare that there is no conflict of interests regarding the publication of this paper.

\section{Acknowledgments}

This work was jointly supported by the National Natural Science Foundation of China (61374080), the Natural Science Foundation of Zhejiang Province (LY12F03010), and a Project Funded by the Priority Academic Program Development of Jiangsu Higher Education Institutions.

\section{References}

[1] V. Andrieu and L. Praly, "A unifying point of view on output feedback designs for global asymptotic stabilization," Automatica, vol. 45, no. 8, pp. 1789-1798, 2009.

[2] H.-B. Du, C.-J. Qian, S.-H. Li, and M. T. Frye, "Global output feedback stabilization of a class of upper-triangular systems with input delay," in Proceedings of the American Control Conference (ACC '12), pp. 5435-5439, Montréal, Canada, June 2012.

[3] D. Karagiannis, A. Astolfi, and R. Ortega, "Two results for adaptive output feedback stabilization of nonlinear systems," Automatica, vol. 39, no. 5, pp. 857-866, 2003.

[4] M.-L. Liu and Y.-G. Liu, "Semi-global stabilization via outputfeedback for a class of uncertain nonlinear systems," Acta Automatica Sinica, vol. 39, no. 12, pp. 2154-2159, 2013.

[5] C.-J. Qian and W. Lin, "Output feedback control of a class of nonlinear systems: a nonseparation principle paradigm," IEEE Transactions on Automatic Control, vol. 47, no. 10, pp. 1710-1715, 2002.

[6] A. Teel and L. Praly, "Tools for semiglobal stabilization by partial state and output feedback," SIAM Journal on Control and Optimization, vol. 33, no. 5, pp. 1443-1488, 1995.

[7] S.-J. Liu and J.-F. Zhang, "Output-feedback control of a class of stochastic nonlinear systems with linearly bounded unmeasurable states," International Journal of Robust and Nonlinear Control, vol. 18, no. 6, pp. 665-687, 2008.

[8] S.-J. Liu, Z.-P. Jiang, and J.-F. Zhang, "Global output-feedback stabilization for a class of stochastic non-minimum-phase nonlinear systems," Automatica, vol. 44, no. 8, pp. 1944-1957, 2008.

[9] W.-S. Chen, J. Wu, and L.-C. Jiao, "State-feedback stabilization for a class of stochastic time-delay nonlinear systems," International Journal of Robust and Nonlinear Control, vol. 22, no. 17, pp. 1921-1937, 2012.

[10] L. Liu and X.-J. Xie, "State feedback stabilization for stochastic feedforward nonlinear systems with time-varying delay," Automatica, vol. 49, no. 4, pp. 936-942, 2013.

[11] L.-C. Guo, X. Zuo, J.-W. Liu, and H.-Q. Liang, "Outputfeedback control of a class of stochastic nonlinear systems with power growth conditions," International Journal of Control, Automation, and Systems, vol. 12, pp. 274-282, 2014.

[12] H. Deng, M. Krstić, and R. J. Williams, "Stabilization of stochastic nonlinear systems driven by noise of unknown covariance," IEEE Transactions on Automatic Control, vol. 46, no. 8, pp. 12371253, 2001.

[13] S.-Y. Khoo, J.-L. Yin, Z.- H. Man, and X.-H. Yu, "Finite-time stabilization of stochastic nonlinear systems in strict-feedback form," Automatica, vol. 49, no. 5, pp. 1403-1410, 2013.
[14] Z.-J. Wu, X.-J. Xie, and S.-Y. Zhang, "Stochastic adaptive backstepping controller design by introducing dynamic signal and changing supply function," International Journal of Control, vol. 79, no. 12, pp. 1635-1646, 2006.

[15] Z.-J. Wu, X.-J. Xie, and S.-Y. Zhang, "Adaptive backstepping controller design using stochastic small-gain theorem," Automatica, vol. 43, no. 4, pp. 608-620, 2007.

[16] X.-J. Xie and L. Liu, "Further results on output feedback stabilization for stochastic high-order nonlinear systems with time-varying delay," Automatica, vol. 48, no. 10, pp. 2577-2586, 2012.

[17] R. Khasminskii, Stochastic Stability of Differential Equations, Springer, New York, NY, USA, 2011.

[18] X.-R. Mao, Stochastic Differential Equations and Applications, Horwood, 2008.

[19] H. Deng and M. Krstić, "Output-feedback stochastic nonlinear stabilization," IEEE Transactions on Automatic Control, vol. 44, no. 2, pp. 328-333, 1999. 


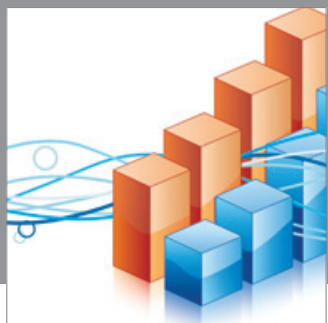

Advances in

Operations Research

mansans

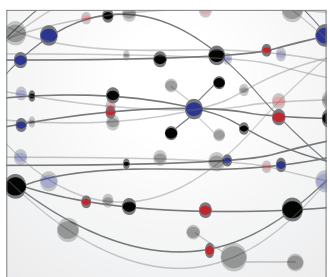

The Scientific World Journal
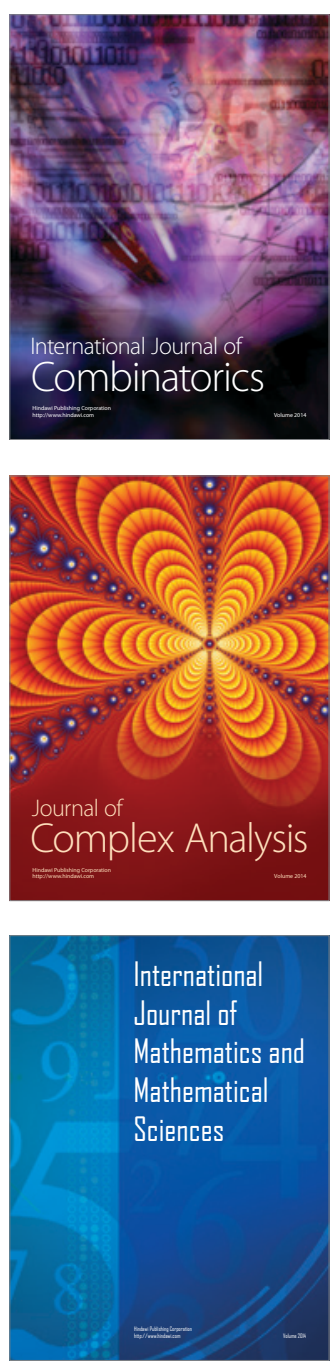
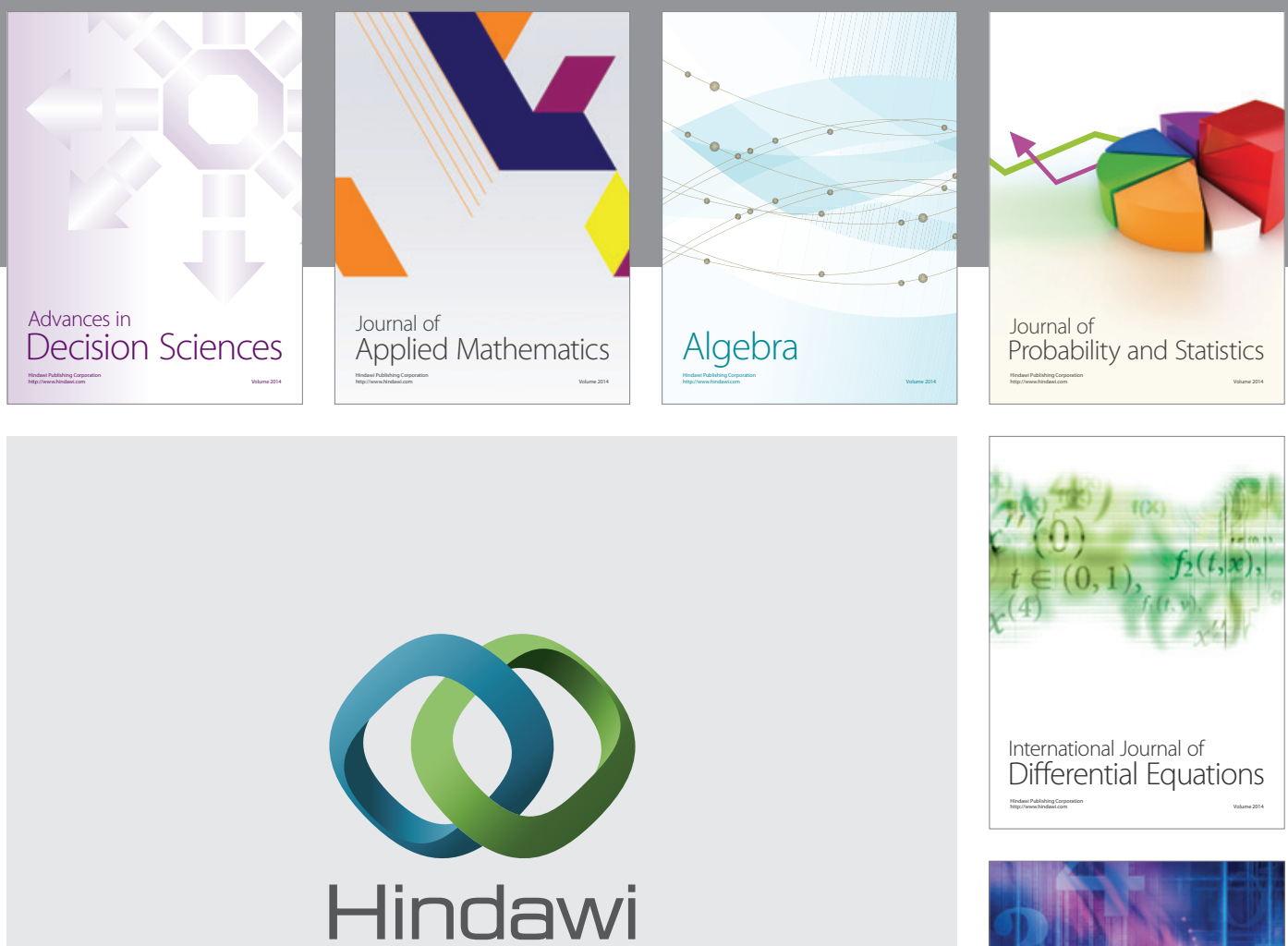

Submit your manuscripts at http://www.hindawi.com
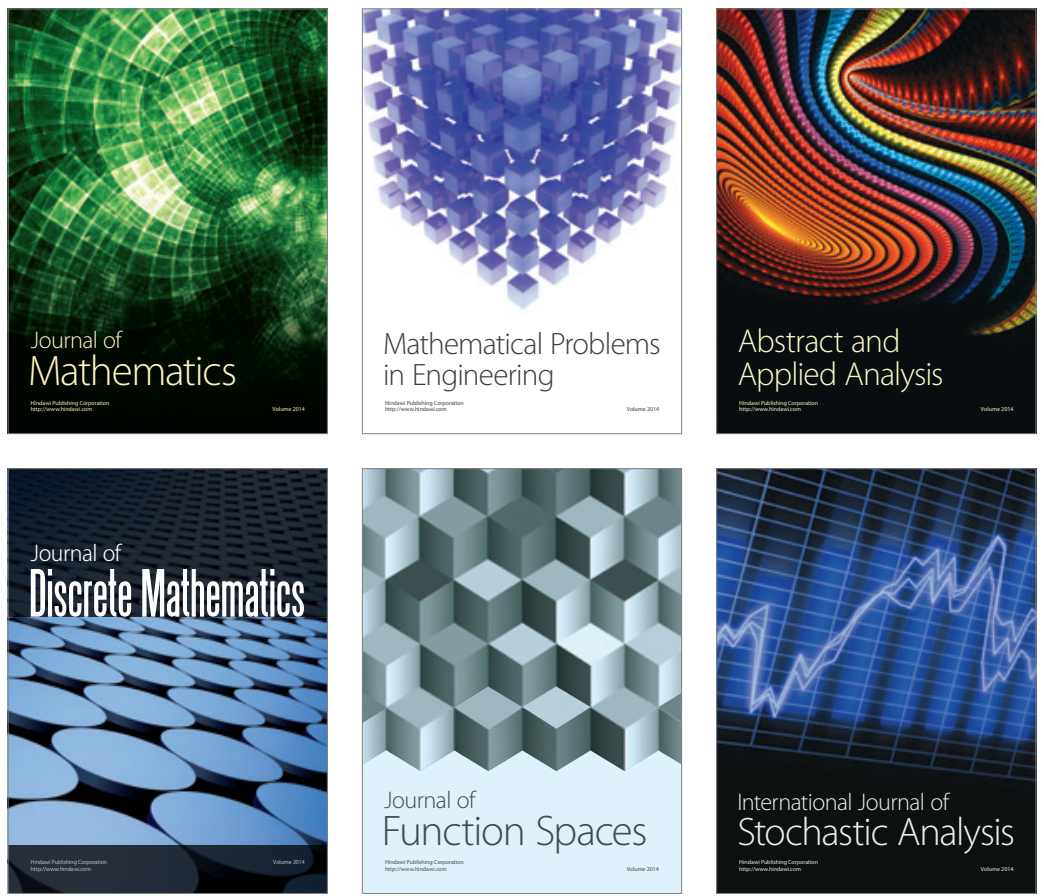

Journal of

Function Spaces

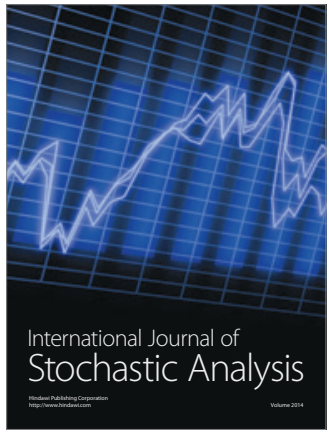

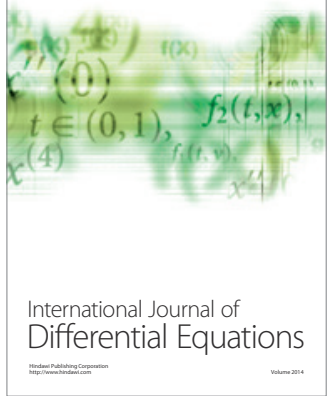
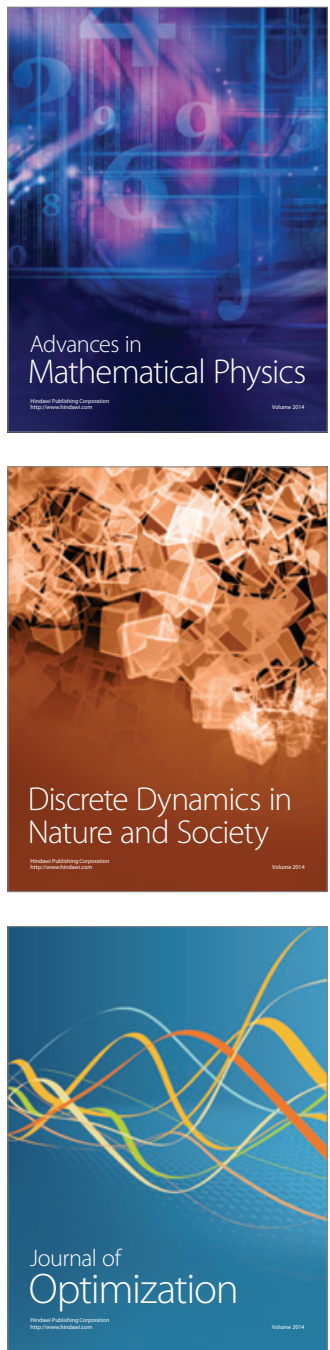